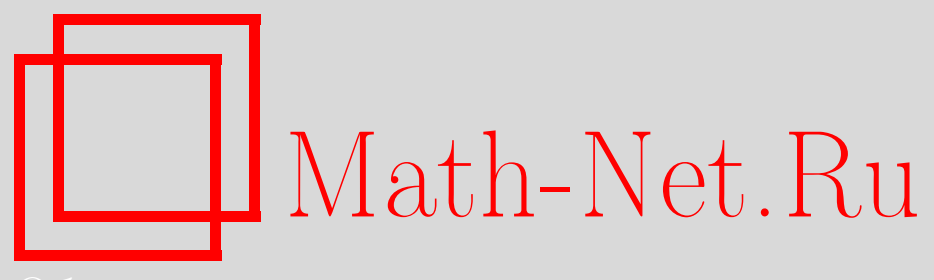

С. С. Мирзоев, А. Р. Алиев, Л. А. Рустамова, Об условиях разрешимости краевой задачи для эллиптического операторно-дифференциального уравнения с разрывным коэффициентом, Матем. заметки, 2012, том 92, выпуск 5, 789-793

DOI: https://doi.org/10.4213/mzm10132

Использование Общероссийского математического портала Math-Net.Ru подразумевает, что вы прочитали и согласны с пользовательским соглашением http: //www. mathnet.ru/rus/agreement

Параметры загрузки:

IP : 3.85 .73 .92

26 апреля 2023 г., 10:30:23

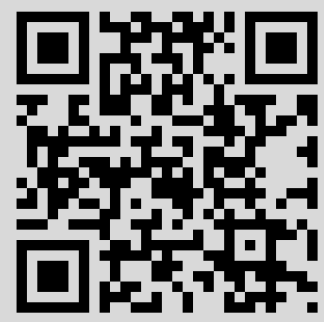




\section{Об условиях разрешимости краевой задачи для эллиптического операторно-дифференциального уравнения с разрывным коэффициентом}

\section{С. С. Мирзоев, А.Р. Алиев, Л. А. Рустамова}

Пусть $H$ - сепарабельное гильбертово пространство и $A$ - самосопряженный равномерно положительный оператор в $H$. Через $H_{\theta}, \theta \geqslant 0$, обозначаем шкалу гильбертовых пространств, порожденных оператором $A$, т.е. $H_{\theta}=\operatorname{Dom}\left(A^{\theta}\right)$ со скалярным произведением $(x, y)_{\theta}=\left(A^{\theta} x, A^{\theta} y\right)$. При $\theta=0$ имеем $H_{0}=H$.

Пусть $L_{2}\left(\mathbb{R}_{+} ; H\right)$ - гильбертово пространство $H$-значных квадратично интегрируемых функций на полуоси $\mathbb{R}_{+}=(0,+\infty)$ (см. [1]), а $W_{2}^{2}\left(\mathbb{R}_{+} ; H\right)$ - пространство $H$-значных функций на $\mathbb{R}_{+}$таких, что $A^{2} u(t)$ и $u^{\prime \prime}(t)$ принадлежат $L_{2}\left(\mathbb{R}_{+} ; H\right)$, причем производные понимаются в смысле теории распределений для функций со значениями в гильбертовом пространстве (см. подробнее [2; гл. 1$])$. Напомним, что нормы в пространствах $L_{2}\left(\mathbb{R}_{+} ; H\right)$ и $W_{2}^{2}\left(\mathbb{R}_{+} ; H\right)$ определяются равенствами

$\|f\|_{L_{2}\left(\mathbb{R}_{+} ; H\right)}=\left(\int_{0}^{+\infty}\|f(t)\|_{H}^{2} d t\right)^{1 / 2}, \quad\|u\|_{W_{2}^{2}\left(\mathbb{R}_{+} ; H\right)}=\left(\left\|A^{2} u\right\|_{L_{2}\left(\mathbb{R}_{+} ; H\right)}^{2}+\left\|u^{\prime \prime}\right\|_{L_{2}\left(\mathbb{R}_{+} ; H\right)}^{2}\right)^{1 / 2}$,

соответственно.

Далее, под $L(X, Y)$ будем понимать множество линейных ограниченных операторов, действующих из гильбертова пространства $X$ в другое гильбертово пространство $Y$. Фиксируем некоторый оператор $K \in L\left(H_{3 / 2}, H_{1 / 2}\right)$. В пространстве $W_{2}^{2}\left(\mathbb{R}_{+} ; H\right)$ рассмотрим следующие подпространства:

$$
\begin{aligned}
\stackrel{\circ}{2}_{2}^{2}\left(\mathbb{R}_{+} ; H\right) & =\left\{u(t): u(t) \in W_{2}^{2}\left(\mathbb{R}_{+} ; H\right), u(0)=u^{\prime}(0)=0\right\}, \\
W_{2, K}^{2}\left(\mathbb{R}_{+} ; H\right) & =\left\{u(t): u(t) \in W_{2}^{2}\left(\mathbb{R}_{+} ; H\right), u^{\prime}(0)=K u(0)\right\} .
\end{aligned}
$$

Тот факт, что $W_{2, K}^{2}\left(\mathbb{R}_{+} ; H\right)$ есть подпространство в $W_{2}^{2}\left(\mathbb{R}_{+} ; H\right)$ следует из теоремы о следах (см. [2; гл. 1]).

Цель заметки - изучение разрешимости задачи

$$
-u^{\prime \prime}(t)+\rho(t) A^{2} u(t)+A_{1} u^{\prime}(t)=f(t), \quad t \in \mathbb{R}_{+},
$$

с краевым условием в нуле

$$
u^{\prime}(0)=K u(0) .
$$

Здесь $f(t) \in L_{2}\left(\mathbb{R}_{+} ; H\right), K \in L\left(H_{3 / 2}, H_{1 / 2}\right), A_{1}$ - линейный оператор такой, что $\operatorname{Dom}\left(A_{1}\right) \supset$ $\operatorname{Dom}(A)$, причем $A_{1} A^{-1}$ ограничен в $H$, а $\rho(t)$ - положительная кусочно постоянная функция с одной точкой разрыва. Для определенности полагаем $\rho(t)=\alpha$, если $t \in(0,1)$, и $\rho(t)=\beta$, если $t \in(1,+\infty)$, причем $0<\alpha \leqslant \beta$.

ОПРЕДЕЛЕниЕ 1. Задача (1), (2) называется регулярно разрешимой, если для каждой функции $f(t) \in L_{2}\left(\mathbb{R}_{+} ; H\right)$ существует функция $u(t) \in W_{2}^{2}\left(\mathbb{R}_{+} ; H\right)$, удовлетворяющая уравнению (1) почти всюду в $\mathbb{R}_{+}$, краевое условие $(2)$ выполняется в смысле сходимости пространства $H_{1 / 2}$, т.е. $\lim _{t \rightarrow 0}\left\|u^{\prime}(t)-K u(t)\right\|_{1 / 2}=0$, и имеет место оценка

$$
\|u\|_{W_{2}^{2}\left(\mathbb{R}_{+} ; H\right)} \leqslant \text { const }\|f\|_{L_{2}\left(\mathbb{R}_{+} ; H\right)} .
$$

Работа выполнена при поддержке Фонда развития науки при Президенте Азербайджанской республики (грант № EIF-2011-1(3)-82/28/1). 
В статье Шкаликова [3] приведены результаты о разрешимости краевых задач для операторно-дифференциальных уравнений общего вида на полуоси и отрезке, а также изучены спектральные проблемы, связанные с разрешимостью таких задач. Но случай, когда коэффициенты в краевых условиях являются не комплексными числами, а операторами, не рассматривался. В [3] приведена также подробная библиография предшествующих работ по этой тематике, среди которых особо выделим работы Гасымова, Костюченко и их учеников. Исследования в этом направлении продолжаются и по сей день (см., например, [4]-[6]). Но работ, посвященных этим вопросам для операторно-значных краевых условий, сравнительно мало. Из числа первых в этой области отметим работы Рофе-Бекетова [7], Ильина и Филиппова [8], Горбачука [9] и более позднюю работу Гасымова и Мирзоева [10]. В последней были изучены как вопросы разрешимости, так и некоторые спектральные проблемы, связанные с краевыми задачами для эллиптических операторно-дифференциальных уравнений второго порядка на полуоси. Основное отличие полученных ниже результатов от работы [10] состоит в том, что функция $\rho(t)$ предполагается разрывной, а не постоянной. Это обстоятельство вносит существенные изменения в метод исследования. Для простоты изложения нами взята одна точка разрыва. Интерес к таким задачам обусловлен тем, что они применимы к широкому кругу задач для дифференциальных уравнений с частными производными и ряду нестандартных задач теории упругости многослойных тел.

Сначала положим в (1) $A_{1}=0$ и рассмотрим более простое уравнение

$$
-u^{\prime \prime}(t)+\rho(t) A^{2} u(t)=f(t), \quad t \in \mathbb{R}_{+} .
$$

Teоpema 1. Пустъ оператор

$$
K_{\alpha, \beta}=E+\frac{1}{\sqrt{\alpha}} A^{-1} K+\frac{\sqrt{\beta}-\sqrt{\alpha}}{\sqrt{\alpha}+\sqrt{\beta}}\left(E-\frac{1}{\sqrt{\alpha}} A^{-1} K\right) e^{-2 \sqrt{\alpha} A}
$$

ограниченно обратим в пространстве $H_{3 / 2}$, где $E$ - единичный оператор в $H$, a $e^{-t A}-$ полугруппа линейных ограниченных операторов, порожденная оператором -A. Тогда оператор $P_{0}$, действующий из пространства $W_{2, K}^{2}\left(\mathbb{R}_{+} ; H\right)$ в пространство $L_{2}\left(\mathbb{R}_{+} ; H\right)$ следующим образом:

$$
P_{0} u(t) \equiv-u^{\prime \prime}(t)+\rho(t) A^{2} u(t), \quad u(t) \in W_{2, K}^{2}\left(\mathbb{R}_{+} ; H\right),
$$

осуществляет изоморфизм между этими пространствами.

Из теоремы 1 следует, что нормы $\left\|P_{0} u\right\|_{L_{2}\left(\mathbb{R}_{+} ; H\right)}$ и $\|u\|_{W_{2}^{2}\left(\mathbb{R}_{+} ; H\right)}$ эквивалентны в пространстве $W_{2, K}^{2}\left(\mathbb{R}_{+} ; H\right)$. Теперь же исследуем краевую задачу $(1),(2)$. Справедлива условная

Теорема 2. Пусть оператор $K_{\alpha, \beta}$ ограниченно обратим в пространстве $H_{3 / 2}$ u оператор $B=A_{1} A^{-1}$ ограничен в $H$, причем $\|B\|<N_{K}^{-1}$, где

$$
N_{K}=\sup _{0 \neq u(t) \in W_{2, K}^{2}\left(\mathbb{R}_{+} ; H\right)}\left\|A u^{\prime}\right\|_{L_{2}\left(\mathbb{R}_{+} ; H\right)}\left\|P_{0} u\right\|_{L_{2}\left(\mathbb{R}_{+} ; H\right)}^{-1} .
$$

Тогда задача (1), (2) регулярно разрешима.

Отметим, что здесь возникает задача об оценке числа $N_{K}$. Воспользуемся работой [11]. Прежде укажем, что имеет место

Лемма 1. При любом $u(t) \in W_{2, K}^{2}\left(\mathbb{R}_{+} ; H\right)$ выполняется неравенство

$$
\begin{aligned}
& \left\|P_{0} u\right\|_{L_{2}\left(\mathbb{R}_{+} ; H\right)}^{2} \\
& \quad \geqslant \frac{\alpha}{\beta}\left(\left\|u^{\prime \prime}\right\|_{L_{2}\left(\mathbb{R}_{+} ; H\right)}^{2}+\alpha \beta\left\|A^{2} u\right\|_{L_{2}\left(\mathbb{R}_{+} ; H\right)}^{2}+2 \beta\left\|A u^{\prime}\right\|_{L_{2}\left(\mathbb{R}_{+} ; H\right)}^{2}+2 \beta \operatorname{Re}\left(A^{-1} K x, x\right)_{3 / 2}\right),
\end{aligned}
$$

где $x=u(0) \in H_{3 / 2}$. 
Для дальнейших действий важную роль играет факторизация рассматриваемого в пространстве $H_{4}$ полиномиального операторного пучка

$$
P(\lambda ; \gamma ; A)=\lambda^{4} E+\alpha \beta A^{4}+(\gamma-2 \beta) \lambda^{2} A^{2} .
$$

Лемма 2. При $\gamma \in\left[0,2 \beta^{1 / 2}\left(\alpha^{1 / 2}+\beta^{1 / 2}\right)\right)$ операторный пучок $P(\lambda ; \gamma ; A)$ обратим на мнимой оси и представляется в виде

$$
P(\lambda ; \gamma ; A)=F(\lambda ; \gamma ; A) F(-\lambda ; \gamma ; A),
$$

где

$$
F(\lambda ; \gamma ; A)=\left(\lambda E-\omega_{1}(\gamma) A\right)\left(\lambda E-\omega_{2}(\gamma) A\right) \equiv \lambda^{2} E+a_{1}(\gamma) \lambda A+a_{2}(\gamma) A^{2},
$$

причем $\operatorname{Re} \omega_{k}(\gamma)<0, k=1,2, a_{1}(\gamma)=\sqrt{2 \beta^{1 / 2}\left(\alpha^{1 / 2}+\beta^{1 / 2}\right)-\gamma}, a_{2}(\gamma)=\alpha^{1 / 2} \beta^{1 / 2}$.

В нашем исследовании не менее особое значение имеет следующее вспомогательное утверждение.

Лемма 3. При любом $u(t) \in W_{2, K}^{2}\left(\mathbb{R}_{+} ; H\right) u \gamma \in\left[0,2 \beta^{1 / 2}\left(\alpha^{1 / 2}+\beta^{1 / 2}\right)\right)$ имеет место тождество

$$
S(u)-\gamma\left\|A u^{\prime}\right\|_{L_{2}\left(\mathbb{R}_{+} ; H\right)}^{2}=\langle R(\gamma) x, x\rangle+\left\|F\left(\frac{d}{d t} ; \gamma ; A\right) u\right\|_{L_{2}\left(\mathbb{R}_{+} ; H\right)}^{2},
$$

где $F(\lambda ; \gamma ; A)$ определен из равенства (4), а

$$
\begin{aligned}
&\langle R(\gamma) x, x\rangle= 2 \beta^{1 / 2}\left(\alpha^{1 / 2}+\beta^{1 / 2}\right) \operatorname{Re}\left(A^{-1} K x, x\right)_{3 / 2} \\
&+\sqrt{2 \beta^{1 / 2}\left(\alpha^{1 / 2}+\beta^{1 / 2}\right)-\gamma}\left(\|x\|_{3 / 2}^{2}+\alpha^{1 / 2} \beta^{1 / 2}\left\|A^{-1} K x\right\|_{3 / 2}^{2}\right), \\
& S(u)=\left\|u^{\prime \prime}\right\|_{L_{2}\left(\mathbb{R}_{+} ; H\right)}^{2}+\alpha \beta\left\|A^{2} u\right\|_{L_{2}\left(\mathbb{R}_{+} ; H\right)}^{2}+2 \beta\left\|A u^{\prime}\right\|_{L_{2}\left(\mathbb{R}_{+} ; H\right)}^{2}+2 \beta \operatorname{Re}\left(A^{-1} K x, x\right)_{3 / 2} .
\end{aligned}
$$

Очевидно, что $S(u)$ есть норма в пространстве $\stackrel{\circ}{W}_{2}^{2}\left(\mathbb{R}_{+} ; H\right)$, эквивалентная исходной норме $\|u\|_{W_{2}^{2}\left(\mathbb{R}_{+} ; H\right)}$.

Выше проведенные исследования позволяют утверждать, что справедлива

Tеорема 3. Число $S_{0}$, определенное как $S_{0}=\sup _{0 \neq u \in \mathfrak{W}_{2}^{2}\left(\mathbb{R}_{+} ; H\right)}\left\|A u^{\prime}\right\|_{L_{2}\left(\mathbb{R}_{+} ; H\right)} S^{-1 / 2}(u)$, конечно и $S_{0}=1 / \sqrt{2 \beta^{1 / 2}\left(\alpha^{1 / 2}+\beta^{1 / 2}\right)}$.

ЗАмечАниЕ 1. Отметим, что, вообще говоря, $S(u)$ не является положительным числом при всех $u(t) \in W_{2, K}^{2}\left(\mathbb{R}_{+} ; H\right)$. Действительно, пусть $u(t)=e^{-\lambda_{0} t} \varphi_{0}$, а $\varphi_{0}$ есть некоторый собственный вектор оператора $A$, отвечающий $\lambda_{0},\left\|\varphi_{0}\right\|=1$. Тогда

$$
\begin{aligned}
S(u) & =\left\|u^{\prime \prime}\right\|_{L_{2}\left(\mathbb{R}_{+} ; H\right)}^{2}+\alpha \beta\left\|A^{2} u\right\|_{L_{2}\left(\mathbb{R}_{+} ; H\right)}^{2}-2 \beta \operatorname{Re}\left(u^{\prime \prime}, A^{2} u\right)_{L_{2}\left(\mathbb{R}_{+} ; H\right)} \\
& =(1+\alpha \beta-2 \beta) \lambda_{0}^{4}\left\|e^{-\lambda_{0} t} \varphi_{0}\right\|_{L_{2}\left(\mathbb{R}_{+} ; H\right)}^{2}=(1+\alpha \beta-2 \beta) \frac{\lambda_{0}^{3}}{2} .
\end{aligned}
$$

Очевидно, что мы можем требовать, чтобы $K=-A$, т.е. условие $K u(0)=u^{\prime}(0)$ выполняется. Тогда ясно, что при $2 \beta-\alpha \beta>1$ выражение $S(u)$ является отрицательным, а при $2 \beta-\alpha \beta=1 S(u)=0, u(t) \in W_{2, K}^{2}\left(\mathbb{R}_{+} ; H\right)$. Поэтому на оператор $K$ необходимо налагать дополнительные условия, чтобы $S(u)$ в пространстве $W_{2, K}^{2}\left(\mathbb{R}_{+} ; H\right)$ было эквивалентно исходной норме $\|u\|_{W_{2}^{2}\left(\mathbb{R}_{+} ; H\right)}$.

Лемма 4. Пусть $x \in H_{3 / 2} . E c л u \min _{\|x\|_{3 / 2}=1}\langle R(0) x, x\rangle>0$, mo $S(u) \geqslant$ const $\|u\|_{W_{2}^{2}\left(\mathbb{R}_{+} ; H\right)}^{2}$ при любом $u(t) \in W_{2, K}^{2}\left(\mathbb{R}_{+} ; H\right)$. 
Из леммы 4 при этом, принимая во внимание теорему о промежуточных производных [2; гл. 1], следует, что

$$
S_{K}=\sup _{0 \neq u \in W_{2, K}^{2}\left(\mathbb{R}_{+} ; H\right)}\left\|A u^{\prime}\right\|_{L_{2}\left(\mathbb{R}_{+} ; H\right)} S^{-1 / 2}(u)<\infty
$$

Поскольку же $W_{2, K}^{2}\left(\mathbb{R}_{+} ; H\right) \supset \stackrel{\circ}{W}_{2}^{2}\left(\mathbb{R}_{+} ; H\right)$, то $S_{K} \geqslant S_{0}=1 / \sqrt{2 \beta^{1 / 2}\left(\alpha^{1 / 2}+\beta^{1 / 2}\right)}$.

Теорема 4. Пусть выполняются условия леммы 4 u $\operatorname{Re} A^{-1} K \geqslant 0$ в $H_{3 / 2}$. Тогда $S_{K}=$ $1 / \sqrt{2 \beta^{1 / 2}\left(\alpha^{1 / 2}+\beta^{1 / 2}\right)}$.

На основании полученных результатов, оценивая норму оператора промежуточной производной $A(d / d t): W_{2, K}^{2}\left(\mathbb{R}_{+} ; H\right) \rightarrow L_{2}\left(\mathbb{R}_{+} ; H\right)$ относительно нормы $\left\|P_{0} u\right\|_{L_{2}\left(\mathbb{R}_{+} ; H\right)}$, при этом учитывая лемму 1 , условная теорема 2 примет точную формулировку в следующем виде.

Теорема 5. Пусть выполняются условия леммы $4, \operatorname{Re} A^{-1} K \geqslant 0$ в $H_{3 / 2}$ и оператор $B=A_{1} A^{-1}$ ограничен в $H$, причем $\|B\|<\sqrt{2 \alpha\left(1+\alpha^{1 / 2} / \beta^{1 / 2}\right)}$. Тогда задача (1), (2) регулярно разрешима.

ЗАмечание 2. В теореме 5 условие $\operatorname{Re} A^{-1} K \geqslant 0$ в $H_{3 / 2}$ обеспечивает обратимость оператора $K_{\alpha, \beta}$ в пространстве $H_{3 / 2}$.

Теперь укажем значение $S_{K}$ при условии $\min _{\|x\|_{3 / 2}=1} \operatorname{Re}\left(A^{-1} K x, x\right)_{3 / 2}<0$.

Теорема 6. Пусть $\min _{\|x\|_{3 / 2}=1} \operatorname{Re}\left(A^{-1} K x, x\right)_{3 / 2}<0$ и выполняются условия леммы 4. Тогда

$S_{K}=\frac{1}{\sqrt{2 \beta^{1 / 2}\left(\alpha^{1 / 2}+\beta^{1 / 2}\right)}}\left(1-2 \beta^{1 / 2}\left(\alpha^{1 / 2}+\beta^{1 / 2}\right)\left|\min _{\|x\|_{3 / 2}=1} \frac{\operatorname{Re}\left(A^{-1} K x, x\right)_{3 / 2}}{1+\alpha^{1 / 2} \beta^{1 / 2}\left\|A^{-1} K x\right\|_{3 / 2}^{2}}\right|^{2}\right)^{-1 / 2}$.

В случае, когда $\min _{\|x\|_{3 / 2}=1} \operatorname{Re}\left(A^{-1} K x, x\right)_{3 / 2}<0$ теорема 2 формулируется следующим образом.

Теорема 7. Пусть выполняются условия теоремы 6 , оператор $K_{\alpha, \beta}$ ограниченно обратим в пространстве $H_{3 / 2}$ и оператор $B=A_{1} A^{-1}$ ограничен в $H$, причем

$$
\|B\|<\sqrt{2 \alpha\left(1+\frac{\alpha^{1 / 2}}{\beta^{1 / 2}}\right)}\left(1-\left.2 \beta^{1 / 2}\left(\alpha^{1 / 2}+\beta^{1 / 2}\right) \min _{\|x\|_{3 / 2}=1} \frac{\operatorname{Re}\left(A^{-1} K x, x\right)_{3 / 2}}{1+\alpha^{1 / 2} \beta^{1 / 2}\left\|A^{-1} K x\right\|_{3 / 2}^{2}}\right|^{2}\right)^{1 / 2} .
$$

Тогда задача (1), (2) регулярно разрешима.

\section{СПИСОК ЦИТИРОВАННОЙ ЛИТЕРАТУРЫ}

[1] Э. Хилле, Р. Филлипс, Функиионалънъй анализ и полугруппы, ИЛ, М., 1962. [2] Ж.-Л. Лионс, Э. Мадженес, Неоднородные граничные задачи и их приложсения, Т. 1, Мир, М., 1971. [3] А. А. Шкаликов, Тр. сем. им. И. Г. Петровского, 14 (1989), 140-224. [4] С. С. Мирзоев, Ф. А. Гулиева, Матем. заметки, 86:5 (2009), 797-800. [5] А. Р. Алиев, С. С. Мирзоев, Функи. анализ и его прил., 44:3 (2010), 63-65. [6] А.Р. Алиев, Матем. заметки, 90:3 (2011), 323-339. [7] Ф. С. Рофе-Бекетов, Матем. сб., 51:3 (1960), 293-342. [8] В. А. Ильин, А. Ф. Филиппов, ДАН СССР, 191:2 (1970), 267-269. [9] М. Л. Горбачук, 
Функи. анализ и его прил., 7:1 (1973), 68-69. [10] М.Г. Гасымов, С. С. Мирзоев, Дифферени. уравнения, 28:4 (1992), 651-661. [11] S.S. Mirzoyev, Trans. Acad. Sci. Azerb. Ser. Phys.-Tech. Math. Sci., 23:1, Math. Mech. (2003), 157-164.

С. С. Мирзоев

Поступило

Бакинский государственный университет,

Институт математики и механики

НАН Азербайджана, г. Баку

E-mail: mirzoyevsabir@mail.ru

\section{А. Р. Алиев}

Бакинский государственный университет,

Институт математики и механики

НАН Азербайджана, г. Баку

E-mail: alievaraz@yahoo.com

\section{Л. А. Рустамова}

Институт прикладной математики

Бакинского государственного университета

E-mail: lamia_rus@rambler.ru 